\title{
PROTÓTIPOS DE SISTEMAS CONSTRUÍDOS DE ÁREAS ALAGADAS PARA TRATAMENTO DE EFLUENTES DE PISCICULTURA
}

\author{
ARCHETYPES OF CONSTRUCTED WETLANDS \\ SYSTEMS FOR TREATMENT OF AQUICULTURE \\ EFFLUENT
}

\section{Monteiro, R.C.M. ${ }^{1}$, Borges, A.K.P. ${ }^{1}$, Tauk-Tornisielo, S.M. ${ }^{1}$; Domingos, R.N. ${ }^{1}$}

${ }^{1}$ Centro Estudos Ambientais, CEA, Universidade Estadual Paulista, UNESP, Campus de Rio Claro, Avenida 24-A, 1515, Bela Vista, CEP 13.506-900, Rio Claro, SP. email: seb@rc.unesp.br

\section{RESUMO}

A piscicultura é uma atividade que acarreta o processo de eutrofização nos corpos de água nos quais seu efluente é lançado, decorrente da nutrição e profilaxias utilizadas em seus tanques de criação. Os compostos que contribuem para o processo de eutrofização são aqueles que contêm nutrientes, como nitrogênio e fósforo. Os manejos utilizados na piscicultura devem ser orientados de tal modo a se evitar o processo de eutrofização, e contemplar tratamentos dos efluentes. Sistemas construídos de áreas alagadas Cosntructed Wetland System - CWS's têm mostrado eficiência quanto ao tratamento de águas, efluentes domésticos e industriais e efluentes de aqüicultura, demonstrando ser um meio eficiente para que ocorram os processos físicos, químicos e biológicos comuns aqueles de áreas alagadas naturais. Foram simulados alguns protótipos de CWS's, em escala de laboratório, utilizando reservatórios de $46 \mathrm{~L}$, com sistema de aeração ativa, fluxo vertical descendente, sendo vegetado com uma policultura de macrófitas aquáticas, para tratamento de efluente de tanques de criação de Oreochromis niloticus (Tilápia do Nilo). Cada par de reservatórios foi preenchido com um dos quatro tipos de matriz suporte: solo de área de cerrado (SC); uma parte de SC para uma parte de cacos de tijolos (agregado); três partes de SC para uma de cacos de tijolos; e somente cacos de tijolos. Os resultados demonstraram que os CWS's contendo SC e o agregado apresentaram estabilização do sistema em um período menor em relação aos demais. Após a estabilização de todos CWS's, as melhores eficiências foram observadas nos 
reservatórios contendo apenas cacos de tijolos ou apenas SC, dependendo do parâmetro analisado.

Palavras-chave: Protótipos. Tratamento de efluentes. Constructed wetland. Piscicultura. Nutrientes

\begin{abstract}
The aquaculture is an activity that finishes causing eutrofization them water bodies in which its effluent one is launched, decurrent of the nutrition and prophylaxes used in its reservoirs. The components that cause greater problems are those that contain nitrogen and phosphorus. The management used in the aquiculture must be guided in such way to prevent the eutrofization and to provide treatment of effluent and water reuse. Constructed wetland system (CWS's) has been efficient for treatment of waters, effluent and domestic and industrial sewer, being pertinent so that physical, chemical and biological processes occur similar as they happen in natural ecosystems. Initially some archetypes of CWS's had been simulated, in laboratory scale, using $46 \mathrm{~L}$ reservoirs, with aeration system, being vegetated with a polyculture of aquatic macrophytes, for effluent treatment of tanks of Tilapia of the Nile creation. The analyzed types of support matrix had been: soil of cerrado area; soil plus brick fragments (1:1) (aggregate); soil plus brick fragments (3:1); and brick fragments. The results had demonstrated that CWS's contend soil plus aggregate was efficient to treat the effluent one used in this study. After the stabilization of CWS's, better efficiencies had been observed in the system only contend brick fragments or only soil, depending on the analyzed parameter.
\end{abstract}

Keywords: Archetypes. Treatment of effluent. Constructed wetland. Aquaculture. Nutrients.

\title{
1. INTRODUÇÃO
}

Os sistemas de áreas alagadas podem ser naturais ou construídos. Os naturais são ecossistemas complexos e fascinantes, que exercem funções vitais para o ambiente e a sociedade, uma vez que sua existência depende da qualidade do meio. Segundo Welsch et al. (1995), as áreas alagadas naturais têm função de regular a água de inundação, detendo as águas provenientes de chuvas intensas (reduzindo os picos de inundação); proteger as margens de lagos e áreas da costa de ações erosivas das ondas e efeitos das chuvas; e promover melhora da qualidade da água, retendo ou transformando o excesso de nutrientes, os sólidos suspensos e metais pesados. Além dessa função de proteção do ambiente, proporciona condições como locais de nidificação, proteção e "habitats" para a vida selvagem. 
Stottmeister et al. (2003) salientaram que os complexos processos proporcionados pelas plantas, microrganismos, o solo e as substâncias presentes na água, juntamente com suas interações ainda não são totalmente compreendidos. $\mathrm{O}$ sistema construído de áreas alagadas (CWS's) pode ser usado isoladamente ou como complemento de outros tipos de tratamentos da água e de efluentes, possibilitando a eficiente remoção de nutrientes, poluentes e de patógenos presentes na água (SHUTES, 2001). Os CWS's distinguem-se dos sistemas naturais porque seus fatores ambientais podem ser controlados e seus processos naturais monitorados (DUARTE, 2002).

Com o aumento da industrialização, das áreas agrícolas e das aqüícolas, ocorrem acréscimos dos descartes de compostos residuais nos sistemas aquáticos, induzindo problemas ambientais e podendo refletir na saúde pública. A qualidade da água verificada mediante suas características físico-químicas e microbiológicas são de fundamental importância para todos os organismos. Alterações na qualidade da água podem comprometer as condições ambientais que propiciam o crescimento e a sobrevivência de espécies da biota aí presente.

A aplicação de tecnologias alternativas pouco onerosas para tratamento de águas poluídas torna-se desejável, em particular, àquelas de fácil manutenção e operação, e que são independentes de energia elétrica. Ansola et al. (2003) citaram a importância de se procurar insistentemente sempre alto nível de performance, principalmente, em relação às remoções de nitrogênio e fósforo. Sistemas de áreas alagadas naturais e construídas têm mostrado alta capacidade de transformar, reciclar, reter e remover diferentes tipos de poluentes, especialmente os nutrientes eutrofizantes, contaminação fecal de esgotos das águas superficiais (HAMMER, 1989) e orgânicos (WALLACE, 1998). Tais sistemas estão baseados nos processos naturais que incluem filtração, sedimentação, adsorção/absorção, biodegradação, que ocorrem nos biofilmes microbianos formados entre a rizosfera e o substrato sólido (TANNER, 1996; GOPAL, 1999).

A aplicação de CWS's tem sido considerada como tecnologia "verde", estimulada pelo menor gasto de energia elétrica em relação a outras tecnologias. Muitos estudos e definições de parâmetros, precisam ser ainda considerados como a sustentabilidade, potencial de reciclagem, capacidade e potencial de tratamento, conservação de ecossistemas e outros. Este processo pode ser considerado um sistema ecológico complexo, sendo inicialmente desenvolvido na América do Norte, por um biologista canadense John Todd (TODD, 1997). O propósito foi demonstrar a possibilidade de se tratar efluente por técnicas ecologicamente viáveis, para reduzir compostos químicos tóxicos, nutrientes e patógenos, podendo ser aplicado na produção de tomates, plantas ornamentais e peixes (BRIX, 1998 apud SHUTES, 2001).

Dentre as águas residuárias que podem ser tratadas com CWS's, pode-se incluir as da piscicultura (MICHAEL, 2003). Nesta pesquisa pretendeu-se investigar a possibilidade de tratar efluentes de pisciculturas, mediante protótipos de CWS's em 
escala de laboratório com fluxo descendente, adequando-os segundo o que recomenda a legislação vigente no país para a classificação das águas, Resolução CONAMA 357 (BRASIL, 2005).

\section{MATERIAL E MÉTODOS}

$\mathrm{Na}$ estufa de vegetação do Centro de Estudos Ambientais (CEA), e em seus laboratórios de Microbiologia e Química, foram construídos os protótipos de CW's, utilizando-se oito caixas plásticas retangulares com capacidade de 46 litros (540mm x $325 \mathrm{~mm}$ largura $\mathrm{x} 390 \mathrm{~mm}$ profundidade). As caixas forma preenchidas com seixos rolados de granulometria média, (cascalho ou pedregulho), obtidos na Mineração Mandu Ltda, do distrito de Ajapí, município de Rio Claro-SP. O tijolo foi obtido na Cerâmica Boa Vista Tomazella Ltda, na Rodovia Washington Luiz Km 188, município de Corumbataí, SP.

O solo usado foi coletado em área de cerrado "sensu stricto", no município de Corumbataí, nas coordenadas entre os meridianos $47^{\circ} 40^{\prime}$ e $47^{\circ} 45^{\prime}$ longitude ocidental e $22^{\circ} 10^{\prime}$ e $22^{\circ} 15^{\prime}$ de latitude sul. Antes da coleta das amostras de solo, a camada de serapilheira foi removida. O solo foi analisado na Faculdade de Ciências Agrárias e Veterinárias, UNESP, Campus Jaboticabal - SP, no Departamento de Solos e Adubos. Apresentou-se como de classe textural média argilosa e $68 \%$ de areia, $4 \%$ de silte e $28 \%$ de argila, $\mathrm{pH}$ de 3,8 e possui $31 \mathrm{~g} / \mathrm{dm} 3$ de matéria orgânica, enquadrando-se como latossolo vermelho amarelo de textura média (fase arenosa). As macrófitas utilizadas foram obtidas na região de Rio Claro, no Centro Nacional de Pesquisa de Peixes Tropicais (CEPTA) do Instituto Brasileiro do Meio Ambiente (IBAMA), município de Pirassununga e na UNESP, Campus Jaboticabal.

Em cada reservatório instalou-se um sistema de drenagem com canos de 1/2 polegada e um sistema de sifão com cano de $200 \mathrm{~mm}$ cortados ao meio, para direcionar o fluxo de água, de modo a passar pelo SC antes da água ser coletada. Sobre o sistema de drenagem foram colocados $5 \mathrm{~cm}$ de seixos rolados e sobre estes $10 \mathrm{~cm}$ do $\mathrm{SC}$, ou $\mathrm{SC}$ e agregado, ou somente agregado (Figura 1). Em todos os reservatórios foram colocadas plantas previamente selecionadas, decorrente dos resultados obtidos em estudos anteriores, que demonstraram maior eficiência do tratamento quando houve associação entre planta e matriz suporte. 


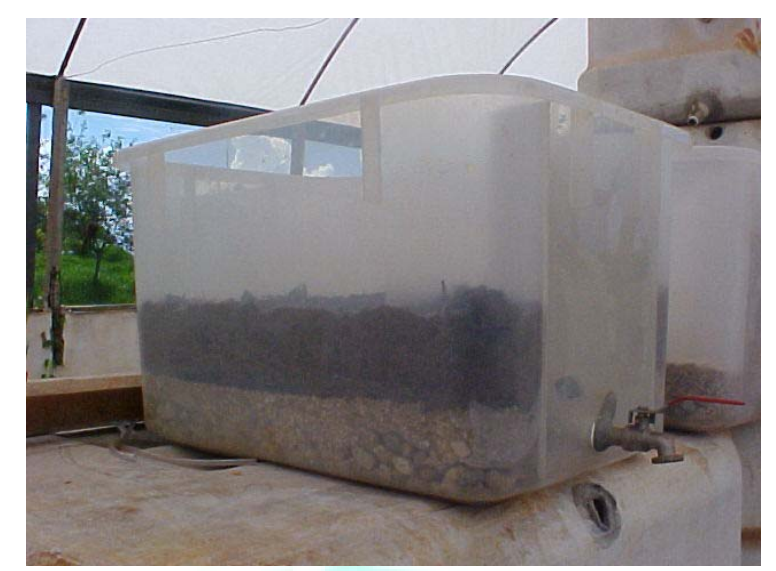

Figura 1. Visão parcial do sistema construído de área alagada mostrando a camada de solo de cerrado e a camada de agregado (cacos de tijolos + pedregulhos).

As plantas empregadas foram em policulturas de: Eichhornia crassipes (aguapé), Salvinia molesta (orelha-de-onça), Pistia stratiotes (alface d'água), Myriophyllum brasiliense (pinheiro d'água), Ludwigia suffruticosa (cruz-de-malta), Heteranthera sp. (aguapé-mirim), Mecardonia procumbens (mecardonia), Eleocharis sp. (cebolinha), Cabomba sp (lodo), Egeria densa (egeria) e Typha sp (taboa). Foram comparados quatro tipos de tratamentos, sendo que a vegetação dos reservatórios foi padronizada. Para cada tratamento foram usadas duas réplicas: 1) cacos de tijolos (Caco); 2) Uma parte de Caco e uma parte de solo filtrante (SC) $(1: 1)$; 3) uma parte de Caco para três partes de SC (1:3); 4) apenas SC. O tempo de retenção hidráulico (T.R.H) foi regulado para 24 horas.

Os oito reservatórios foram preenchidos até saturação com efluente de tanque usado para criação de tilápias e a primeira amostragem do efluente foi realizada no dia seguinte após a montagem do sistema. Foram utilizadas duas caixas de $1.000 \mathrm{~L}$ para simulação de piscicultura usando 40 tilápias, instaladas antes do início deste experimento, para que seu efluente se aproximasse daquele produzido na escala real dos pesqueiros.

As amostras dos efluentes foram realizadas nos tempos de $1 ; 8 ; 15 ; 30$ e 51 dias e coletadas nas saídas de cada reservatório e das caixas usadas para simulação de piscicultura (efluente). As coletas foram realizadas sempre no mesmo horário (9:00 h) e imediatamente processadas, com exceção do nitrogênio total. Para gerar efluente "fresco", foi estruturada uma mini piscicultura de tilápias do Nilo. Os parâmetros estudados foram escolhidos por serem os mais usuais, mas também por indicarem melhor a eficiência dos CWS's, mesmo em escala de laboratório.

Os parâmetros analisados foram: temperatura do ar $\left({ }^{\circ} \mathrm{C}\right)$ com termômetro de mercúrio em vidro da marca I.M.Total; temperatura da água $\left({ }^{\circ} \mathrm{C}\right)$ e $\mathrm{pH}$ no aparelho de campo Mettler Toledo, modelo MP 120; condutividade elétrica ( $\mu \mathrm{S} . \mathrm{cm}-1)$, salinidade e sólidos totais dissolvidos (STD) (mg. $\left.\mathrm{L}^{-1}\right)$ através do aparelho de campo Hach, modelo CO 150; oxigênio dissolvido (OD) $\left(\mathrm{mg} . \mathrm{O}_{2} \cdot \mathrm{L}^{-1}\right)$ no aparelho de campo YSI 556 MPS; cor aparente $(\mathrm{Pt} / \mathrm{L})$ em aparelho Hach modelo DR/2000; turbidez (UNT) usando equipamento Hach, modelo 2100P; nitrogênio amoniacal $\left(\mu \mathrm{g} . \mathrm{L}^{-1}\right)$, nitrato, 
nitrito, nitrogênio total (mg.. $\left.\mathrm{L}^{-1}\right)$ com o método de Mackereth, Heron e Talling (1978); fósforo total $\left(\mu \mathrm{g} \cdot \mathrm{L}^{-1}\right)$ com método de Golderman, Clymo e Ohnstand (1978); coliformes totais e Escherichia coli usando Kit Colilert ${ }^{\circledR}$ IDEXX Laboratories Inc. Westbook, ME (foram analisados nos dias 30 e 51) e a demanda bioquímica de oxigênio (DBO) (mg. $\left.\mathrm{L}^{-1}\right)$ em aparelho Hach DBO Track e demanda química de oxigênio (DQO) (mg. $\mathrm{L}^{-1}$ ) em aparelho Hach, modelo DR/2000 e COD Reacto (no 51dia).

Os resultados obtidos foram representados através de suas médias, com exceção do $\mathrm{pH}$. As eficiências dos sistemas foram calculadas a partir da seguinte fórmula: Eficiência $(\%)=[($ Valor Efluente - Valor Após Tratamento $) \times$ Valor Efluente-1] x 100. Os resultados quando positivos representaram a ocorrência de eficiência do processo e quando negativos, expressaram a ineficiência deste mesmo processo.

\section{RESULTADOS E DISCUSSÃO}

Os parâmetros estudados foram escolhidos por serem os mais usuais, mas também por indicarem melhor a eficiência dos CWS's, mesmo em escala de laboratório. As médias dos resultados e eficiências estão relacionadas nas tabelas 1 a 5 .

Os valores da condutividade elétrica $\left(\mu \mathrm{S} / \mathrm{cm}^{2}\right)$ situaram entre 155,5 e 404,0 (Tabelas 1 a 5), nos diferentes sistemas aqui usados, sendo que a maior diminuição dos mesmos ocorreu após 30 dias no sistema contendo apenas SC (43,2\%). No final do experimento, 51 dias, os valores deste parâmetro bem como as eficiências de cada tratamento estiveram muito próximas. Nos tratamentos contendo apenas cacos de tijolos e naquele contendo cacos e SC (1:1), houve aumento da eficiência, que inicialmente foi intensificada pelas maiores quantidades de SC na matriz suporte, agindo como um catalisador.

Tabela 1 - Médias dos valores e das eficiências dos parâmetros determinados nos tratamentos em CWs após um dia de tratamento.

\begin{tabular}{|c|c|c|c|c|c|c|c|c|c|}
\hline \multicolumn{10}{|c|}{ Médias e Eficiências (\%) Referentes a um dia de tratamento } \\
\hline Temperatura do Ar $19,5^{\circ} \mathrm{C}$ & Cacos & $\%$ & $1 \mathrm{C} / 1 \mathrm{SC}$ & $\%$ & $1 \mathrm{C} / 3 \mathrm{SC}$ & $\%$ & $\mathrm{SC}$ & $\%$ & Efluente \\
\hline Temperatura da Água ${ }^{\circ} \mathrm{C}$ & 14,6 & & 14,6 & & 14,7 & & 14,7 & & 18,3 \\
\hline $\mathrm{pH}$ & $8,1-8,0$ & & $5,8-5,9$ & & $5,5-5,4$ & & $6,7-6,3$ & & 8,4 \\
\hline Condutividade Elétrica uScm ${ }^{2-1}$ & 404,0 & 9,2 & 331,0 & 25,6 & 317,5 & 28,7 & 344,0 & 22,7 & 445,0 \\
\hline Salinidade \% & 0,2 & 7,1 & 0,2 & 23,8 & 0,2 & 26,2 & 0,2 & 21,4 & 0,2 \\
\hline Sólidos Totais Dissolvidos g. $\mathrm{L}^{-1}$ & 0,22 & 24,6 & 0,22 & 25,3 & 0,21 & 28,4 & 0,22 & 22,7 & 0,29 \\
\hline Oxigênio Dissolvido mg. $\mathrm{L}^{-1}$ & 9,2 & 4,1 & 7,7 & $-12,9$ & 7,0 & $-21,2$ & 9,0 & 2,2 & 8,8 \\
\hline $\mathrm{DO} \%$ & 90,4 & $-3,9$ & 75,6 & $-19,7$ & 68,6 & $-27,1$ & 89,1 & $-5,4$ & 94,1 \\
\hline Cor PtCo & 75,0 & 42,3 & 49,0 & 62,7 & 48 & 63,5 & 95 & 27,3 & 130,0 \\
\hline Turbidez UNT & 5,7 & 44,0 & 3,5 & 66,0 & 5,3 & 48,4 & 7,1 & 30,0 & 10,2 \\
\hline Amônia mg. $\mathrm{L}^{-1}$ & 0,3 & 30,6 & 2,3 & $-544,4$ & 4,5 & $-1136,1$ & 4,2 & $-1052,8$ & 0,4 \\
\hline Nitrito mg. $\mathrm{L}^{-1}$ & 0,28 & 72,4 & 0,18 & 82,2 & 0,15 & 85,5 & 0,22 & 78,9 & 1,03 \\
\hline Nitrato $\mathrm{mg} \cdot \mathrm{L}^{-1}$ & 1,2 & 40,0 & 2,6 & $-27,5$ & 3,6 & $-80,0$ & 2,8 & $-37,5$ & 2,0 \\
\hline Nitrogênio Total mg. $\mathrm{L}^{-1}$ & 0,8 & 33,3 & 3,4 & $-172,8$ & 5,5 & $-338,9$ & 5,5 & $-333,3$ & 1,3 \\
\hline Fósforo Total $\mu \mathrm{g} . \mathrm{L}^{-1}$ & 80,1 & 82,1 & 29,7 & 93,4 & 33,4 & 92,5 & 44,6 & 90,0 & 446,9 \\
\hline
\end{tabular}


Tabela 2 - Médias dos valores e das eficiências dos parâmetros determinados nos tratamentos em CWs após oito dias de tratamento.

\begin{tabular}{|c|c|c|c|c|c|c|c|c|c|}
\hline \multicolumn{10}{|c|}{ Médias e Eficiências Referentes a 8 dias de tratamento } \\
\hline Temperatura do Ar $19,5^{\circ} \mathrm{C}$ & Caco & $\%$ & $1 \mathrm{C} / 1 \mathrm{SC}$ & $\%$ & $1 \mathrm{C} / 3 \mathrm{SC}$ & $\%$ & $\mathrm{SC}$ & $\%$ & Efluente \\
\hline Temperatura da Água ${ }^{\circ} \mathrm{C}$ & 12,4 & & 12,6 & & 12,1 & & 12,1 & & 18,4 \\
\hline $\mathrm{pH}$ & $7,4-7,0$ & & $5,9-6,1$ & & $5,1-4,9$ & & $5,2-5,1$ & & 8,0 \\
\hline Condutividade Elétrica $\mathrm{uScm}^{2-1}$ & 381,5 & $-0,1$ & 322,5 & 15,4 & 304,0 & 20,2 & 314,0 & 17,6 & 381,0 \\
\hline Salinidade \% & 0,2 & 0,0 & 0,2 & 13,9 & 0,2 & 16,7 & 0,2 & 16,7 & 0,2 \\
\hline Sólidos Totais Dissolvidos g. $\mathrm{L}^{-1}$ & 0,25 & 0,0 & 0,21 & 14,9 & 0,2 & 20,4 & 0,20 & 17,5 & 0,25 \\
\hline Oxigênio Dissolvido mg. $\mathrm{L}^{-1}$ & 4,8 & 26,1 & 3,7 & $-3,9$ & 2,5 & $-34,6$ & 2,0 & $-46,9$ & 3,8 \\
\hline $\mathrm{DO} \%$ & 45,3 & 10,1 & 34,5 & $-16,1$ & 23,4 & $-43,2$ & 19,0 & $-53,9$ & 41,1 \\
\hline Cor PtCo & 39,0 & 79,2 & 39,0 & 78,9 & 29,0 & 84,3 & 45,0 & 75,7 & 185 \\
\hline Turbidez UNT & 2,2 & 88,4 & 1,8 & 90,2 & 2,0 & 89,3 & 2,3 & 87,7 & 18,7 \\
\hline Amônia mg. $\mathrm{L}^{-1}$ & 0,1 & 64,7 & 0,4 & $-2,9$ & 0,8 & $-122,1$ & 2,6 & $-669,1$ & 0,3 \\
\hline Nitrito mg. $\mathrm{L}^{-1}$ & 0,00 & 76,3 & 0,01 & 65,8 & 0,00 & 84,2 & 0,00 & 84,2 & 0,02 \\
\hline Nitrato $\mathrm{mg} . \mathrm{L}^{-1}$ & 0,3 & $-50,0$ & 0,2 & 0,0 & 0,2 & 25,0 & 0,2 & 0,0 & 0,2 \\
\hline Nitrogênio Total mg. $\mathrm{L}^{-1}$ & 0,6 & 73,3 & 0,6 & 73,3 & 1,2 & 43,0 & 3,7 & $-76,7$ & 2,1 \\
\hline Fósforo Total $\mu \mathrm{g} . \mathrm{L}^{-1}$ & 168,4 & 68,1 & 28,3 & 94,6 & 12,1 & 97,7 & 26,0 & 95,1 & 528,4 \\
\hline
\end{tabular}

Quanto aos valores de STD (g. $\left.\mathrm{L}^{-1}\right)$, estes variaram entre 0,1 e 0,2 (Tabelas 1 a 5). Os desempenhos dos diferentes sistemas utilizados foram semelhantes àqueles observados para a condutividade elétrica.

A cor aparente variou entre 29 e 252 PtCo (Tabelas 1 a 5), tendo maiores valores após 30 dias e os menores aos 15 dias de tratamento. Os valores obtidos de turbidez e da cor aparente tiveram comportamento semelhante. Os maiores e menores valores da turbidez ficaram entre 1,8 e 10,0UNT. O efluente após o tratamento enquadrou-se dentro das características de águas de classe 1, segundo a Resolução CONAMA 357 (BRASIL, 2005). Em relação a este parâmetro os CWS's estudados apresentaram as maiores eficiências entre 8 e 15 dias de tratamento.

Tabela 3 - Médias dos valores e das eficiências dos parâmetros determinados nos tratamentos em CWs após quinze dias de tratamento.

\begin{tabular}{|c|c|c|c|c|c|c|c|c|c|}
\hline \multicolumn{10}{|c|}{ Médias e Eficiências Referentes a 15 de tratamento } \\
\hline Temperatura do $\mathrm{Ar} 17,0^{\circ} \mathrm{C}$ & Caco & $\%$ & $1 \mathrm{C} / 1 \mathrm{SC}$ & $\%$ & $1 \mathrm{C} / 3 \mathrm{SC}$ & $\%$ & $\mathrm{SC}$ & $\%$ & Efluente \\
\hline Temperatura da Água ${ }^{\circ} \mathrm{C}$ & 16,9 & & 16,9 & & 16,9 & & 16,8 & & 22,7 \\
\hline $\mathrm{pH}$ & $7,7-6,1$ & & $6,9-6,1$ & & $5,6-5,0$ & & 5,1 & & 7,4 \\
\hline Condutividade Elétrica uScm ${ }^{2-1}$ & 351,5 & 5,5 & 311,0 & 16,4 & 255,0 & 31,5 & 283,0 & 23,9 & 372,0 \\
\hline Salinidade \% & 0,2 & 5,6 & 0,2 & 16,7 & 0,1 & 33,3 & 0,1 & 37,5 & 0,2 \\
\hline Sólidos Totais Dissolvidos g...-1 & 0,23 & 5,6 & 0,20 & 16,3 & 0,17 & 31,6 & 0,18 & 24,2 & 0,24 \\
\hline Oxigênio Dissolvido mg. $\mathrm{L}^{-1}$ & 4,3 & 51,8 & 2,8 & $-1,2$ & 2,9 & 1,8 & 2,7 & $-6,0$ & 2,8 \\
\hline $\mathrm{DO} \%$ & 44,1 & 34,9 & 28,8 & $-12,1$ & 29,7 & $-9,3$ & 27,3 & $-16,7$ & 32,7 \\
\hline Cor PtCo & 31,0 & 87,8 & 63,0 & 75,3 & 47,0 & 81,8 & 67,0 & 73,7 & 255 \\
\hline Turbidez UNT & 1,8 & 92,4 & 2,9 & 87,7 & 1,9 & 91,9 & 2,4 & 89,7 & 23,2 \\
\hline Amônia mg. $\mathrm{L}^{-1}$ & 0,1 & 76,0 & 0,3 & 42,3 & 0,5 & 1,9 & 1,2 & $-125,0$ & 0,5 \\
\hline Nitrito $\mathrm{mg} \cdot \mathrm{L}^{-1}$ & 0,00 & 85,2 & 0,01 & 77,8 & 0,00 & 83,3 & 0,00 & 88,9 & 0,03 \\
\hline Nitrato $\mathrm{mg} \cdot \mathrm{L}^{-1}$ & 0,2 & 25,0 & 0,1 & 50,0 & 0,0 & 100,0 & 0,1 & 50,0 & 0,2 \\
\hline Nitrogênio Total mg. $\mathrm{L}^{-1}$ & 0,6 & 55,6 & 0,8 & 33,3 & 0,9 & 27,8 & 1,7 & $-36,1$ & 1,3 \\
\hline Fósforo Total $\mu \mathrm{g} . \mathrm{L}^{-1}$ & 97,7 & 87,4 & 24,5 & 96,8 & 7,2 & 99,1 & 4,9 & 99,4 & 776,5 \\
\hline
\end{tabular}


Para cor aparente e turbidez, os valores não foram distintos nos diferentes tratamentos, todavia o sistema contendo apenas cacos de tijolos mostrou melhores resultados na remoção dos mesmos e o uso do agregado possibilitou melhor desempenho do SC durante o tratamento. Os resultados de salinidade apresentaram valores que não ultrapassaram a $0,2 \%$ (Tabelas 1 a 5), portanto, inferiores ao máximo permitido para as águas doces $(0,5 \%)$. Nos sistemas estudados, as possíveis alterações da salinidade observadas foram, provavelmente, decorrentes do uso de sal grosso $(\mathrm{NaCl})$ no manejo dos peixes objetivando amenizar o "stress" causado durante alguma prática.

Tabela 4 - Médias dos valores e das eficiências dos parâmetros determinados nos tratamentos em CWs após trinta dias de tratamento.

\begin{tabular}{|c|c|c|c|c|c|c|c|c|c|}
\hline \multicolumn{10}{|c|}{ Médias e Eficiências Referentes a 30 dias de tratamento } \\
\hline Temperatura do $\mathrm{Ar} 20,2^{\circ} \mathrm{C}$ & Caco & $\%$ & $1 \mathrm{C} / 1 \mathrm{SC}$ & $\%$ & $1 \mathrm{C} / 3 \mathrm{SC}$ & $\%$ & $\mathrm{SC}$ & $\%$ & Efluente \\
\hline Temperatura da Água ${ }^{\circ} \mathrm{C}$ & 18,6 & & 19,5 & & 20,0 & & 19,9 & & 24,5 \\
\hline $\mathrm{pH}$ & $7,7-7,6$ & & $8,3-7,2$ & & $7,6-6,5$ & & $4,9-5,3$ & & 7,5 \\
\hline Condutividade Elétrica uScm ${ }^{2-1}$ & 258,0 & 5,8 & 252,5 & 7,8 & 208,0 & 24,1 & 155,5 & 43,2 & 274,0 \\
\hline Salinidade \% & 0,1 & 7,7 & 0,1 & 7,7 & 0,1 & 23,1 & 0,1 & 42,3 & 0,1 \\
\hline Sólidos Totais Dissolvidos g. $\mathrm{L}^{-1}$ & 0,168 & 5,9 & 0,16 & 7,9 & 0,14 & 23,3 & 0,10 & 43,0 & 0,18 \\
\hline Oxigênio Dissolvido mg. $\mathrm{L}^{-1}$ & 7,1 & 63,6 & 9,4 & 116,4 & 8,4 & 93,1 & 5,5 & 26,7 & 4,3 \\
\hline $\mathrm{DO} \%$ & 75,9 & 45,6 & 105,0 & 101,5 & 92,6 & 77,6 & 60,1 & 15,4 & 52,1 \\
\hline Cor PtCo & 91,0 & 75,2 & 145,0 & 60,3 & 182 & 50,3 & 220 & 39,7 & 365 \\
\hline Turbidez UNT & 5,1 & 78,7 & 7,9 & 67,1 & 8,6 & 64,0 & 10,0 & 58,0 & 23,9 \\
\hline Amônia mg. $\mathrm{L}^{-1}$ & 0,2 & 73,8 & 0,4 & 58,1 & 0,5 & 36,6 & 1,0 & $-14,5$ & 0,9 \\
\hline Nitrito mg. $\mathrm{L}^{-1}$ & 0,01 & 89,4 & 0,01 & 86,9 & 0,01 & 85,0 & 0,01 & 90,0 & 0,08 \\
\hline Nitrato $\mathrm{mg} \cdot \mathrm{L}^{-1}$ & 0,2 & 66,7 & 0,4 & 33,3 & 0,6 & 8,3 & 0,8 & $-33,3$ & 0,6 \\
\hline Nitrogênio Total mg. $\mathrm{L}^{-1}$ & 0,1 & 97,6 & 1,8 & 70,2 & 2,4 & 59,5 & 2,2 & 63,1 & 5,9 \\
\hline Fósforo Total $\mu \mathrm{g} . \mathrm{L}^{-1}$ & 178,7 & 73,1 & 124,1 & 81,4 & 178,3 & 73,2 & 79,0 & 88,1 & 665,3 \\
\hline Coliformes Totais NMP/100mL & 7701,0 & 94,6 & 1153,0 & 99,2 & 15531,0 & 89,0 & 24192,0 & 82,9 & 141360,0 \\
\hline Escherichia coli NMP/100mL & 0 & & 0 & & 0 & & 0 & & 0 \\
\hline
\end{tabular}

Tabela 5 - Médias dos valores e das eficiências dos parâmetros determinados nos tratamentos em CWs após cinqüenta e um dias de tratamento.

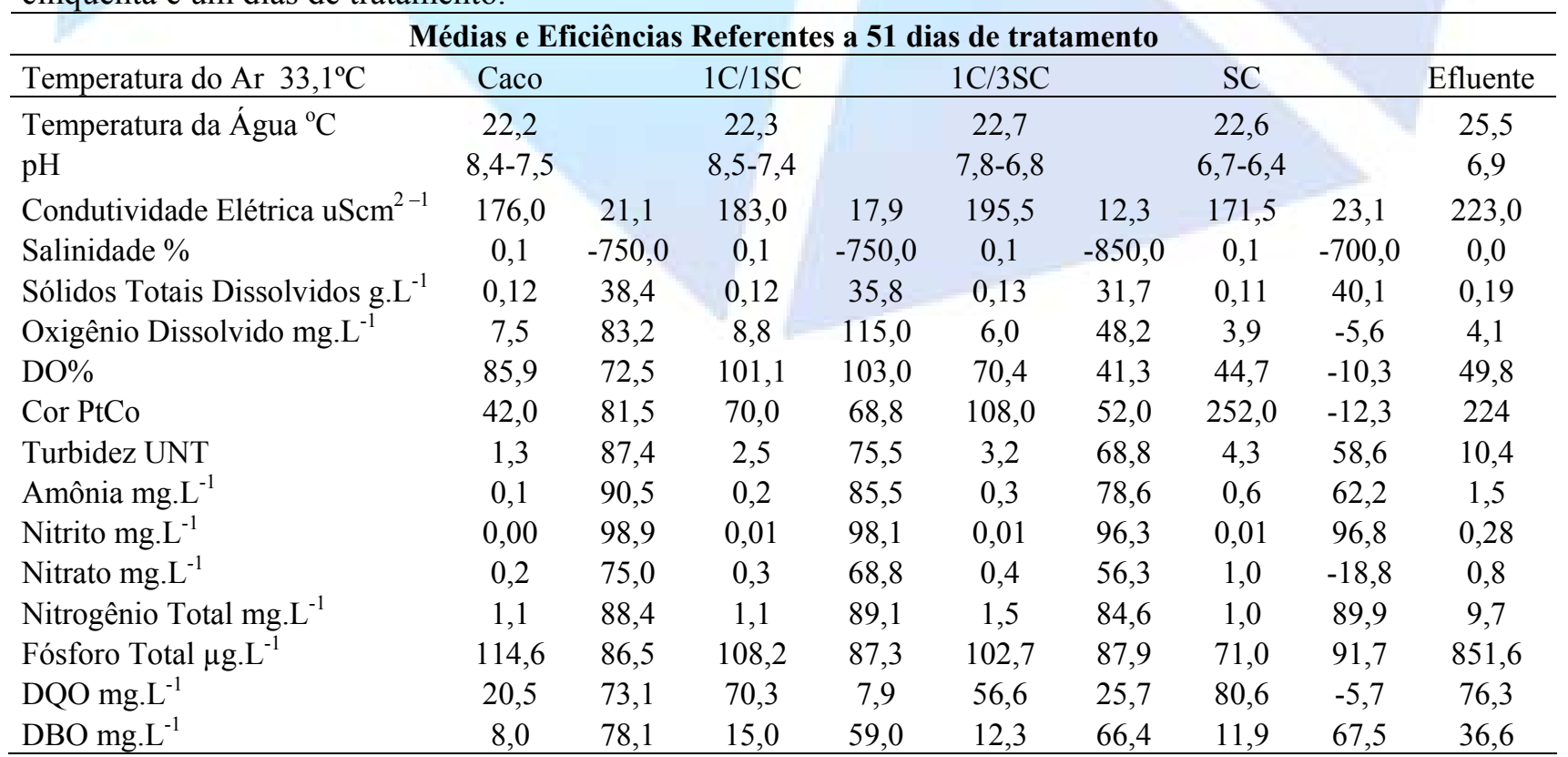


Os resultados de $\mathrm{pH}$ indicaram que nos CWS's com SC houve tendência ao decréscimo de seus valores, ao contrário do observado no tratamento com apenas cacos de tijolos. A acidificação natural do SC foi inibida pelo uso do agregado. Os valores de $\mathrm{OD}$, cor aparente, turbidez e nitrito acompanharam as variações ocorridas no efluente, sendo que maiores valores médios obtidos ocorreram aos 30 dias de tratamento (Tabela 4) e os menores entre 8 e 15 dias (Tabelas 2 e 3). Segundo a Resolução CONAMA 357 (BRASIL, 2005), a água de rio de classe 1 deve possuir no mínimo de $6 \mathrm{mg}^{-\mathrm{L}^{-1}}$ de OD, quantidade observada no CWS's contendo apenas cacos de tijolos, após 22 dias do inicio do experimento.

O oxigênio é um fator limitante no ciclo do nitrogênio, tendo grande importância para o crescimento de bactérias responsáveis pela amonificação e nitrificação. Com exceção do CWS's com apenas cacos de tijolos, observou-se que o consumo inicial do oxigênio pode ser decorrente da decomposição da matéria orgânica que estava presente no SC $\left(31 \mathrm{~g} \cdot \mathrm{dm}^{3-1}\right)$. A oxigenação da água pode ter sido resultante do uso de Egeria densa e Cabomba sp, sendo possível verificar bolhas de ar na superfície de suas folhas.

No tratamento com apenas cacos de tijolos, os valores de OD não sofreram mudanças bruscas e o sistema mostrou-se progressivamente mais eficiente a cada amostragem.

Com o decorrer do experimento, todos os tratamentos apresentaram diminuição da concentração de amônia, sendo que a adição de cacos de tijolos ao SC possibilitou reduções iniciais e finais mais rápidas (proporcional à quantidade adicionada). Os valores iniciais elevados devem estar relacionados com a decomposição da matéria orgânica presente no solo. Os valores da amônia ficaram entre 0,1 e 4,5mg/L (Tabelas 1 a 5).

O nitrito é uma forma intermediária e instável do nitrogênio e sua concentração no meio geralmente é baixa. Em ambientes oxigenados, o nitrito é oxidado a nitrato, e em ambiente com pouco oxigênio, possibilita a formação de amônia. Todos os CWS's possibilitaram diminuição deste parâmetro durante o experimento, sendo que a eficiência aumentou conforme o decorrer do tempo do experimento . Os valores ficaram entre 0,00 e $0,28 \mathrm{mg} \cdot \mathrm{L}^{-1}$ (Tabelas 1 a 5) e a presença de SC, inicialmente, possibilitou melhores remoções, superadas com maior tempo de tratamento em CWS's contendo apenas cacos de tijolos.

A remoção do nitrato teve grande variação entre os sistemas aqui utilizados. Pode-se observar uma relação entre os valores encontrados no efluente e naquele após o tratamento em CWS's. A remoção deste parâmetro demonstrou tendência de estabilização com o decorrer do tempo de tratamento, sendo que a maior eficiência de remoção ocorreu no CWS's com apenas cacos de tijolos. Os valores do nitrato entre 0,0 e 3,6mg. $\mathrm{L}^{-1}$ (Tabelas 1 a 5) foram verificados no CWS's contendo três partes SC para uma de cacos de tijolos (3:1). As concentrações observadas de nitrogênio total foram baixas neste experimento, contudo, foi observada diminuição das mesmas, inicialmente apenas no tratamento de cacos de tijolos, fato que está relacionado com 
o aumento da amônia nos tratamentos contendo SC. Nestes, verificou-se que maior quantidade de solo acarretou acréscimo mais lento da eficiência do tratamento.

No final do experimento, as remoções atingiram 84,6 e 89,9\%, estas consideradas satisfatórias para a liberação do efluente tratado em qualquer curso de água. Os valores do nitrogênio total situaram-se entre 0,1 e 5,5mg.L $\mathrm{L}^{-1}$ (Tabelas 1 a 5) sendo que os valores mais elevados foram obtidos na primeira coleta (Tabela 1) para $\mathrm{SC}$ e para o sistema contendo três partes de SC e uma de cacos de tijolos (3:1), que também apresentaram os maiores valores de amônia.

O fósforo foi removido com eficiência nos sistemas, principalmente naqueles contendo solo, sendo que maiores quantidades deste proporcionaram melhores reduções dos valores deste parâmetro. Os valores do fósforo ficaram entre 4,9 e $178,7 \mu \mathrm{g} . \mathrm{L}^{-1}$ (Tabelas 1 a 5). A maior eficiência do uso do SC talvez se deva a capacidade de adsorção do fósforo, processo intensificado pela presença de compostos orgânicos, bem como pela presença de alumínio e ferro. Com o decorrer do experimento, os diferentes CWS's mostraram eficiências semelhantes.

Não foi constatada a presença de Escherichia coli no efluente. A redução de coliformes totais foi obtida em todos os sistemas, sendo que a associação de SC e cacos de tijolos (1:1) possibilitou melhor eficiência do sistema, reduzindo em 99,2\% o número mais provável (NMP) dessas bactérias. Elias (2003) encontrou eficiência de $99,5 \%$ na redução de coliformes totais e os valores de coliformes totais ficaram entre 1.153 e 24.192 NMP. $100 \mathrm{~mL}^{-1}$. Nestes experimentos não foi possível identificar a ação da radiação solar na eliminação dos patógenos, uma vez que os mesmos foram realizados em estufa de vegetação.

A redução da DBO foi observada em todos CWS's, sendo superior naquele contendo apenas cacos de tijolos e os valores situaram entre 8,0 e $15,0 \mathrm{mg} . \mathrm{L}^{-1}$ (Tabelas 1 a 5). O CWS's contendo apenas cacos possibilitou também a maior eficiência na redução da $\mathrm{DQO}$, contudo, os demais tratamentos não acarretaram eficiências semelhantes àquelas verificadas para a $\mathrm{DBO}$. A presença de $\mathrm{SC}$ no sistema não acarretou diminuição dos valores de $\mathrm{DQO}$, ao contrário, induziu aumento dos valores deste parâmetro que permaneceram entre 20,5 e $80,6 \mathrm{mg} \cdot \mathrm{L}^{-1}$.

As eficiências máximas foram encontradas apenas após 15 dias de tratamento, isto sendo observados em experimentos preliminares deste estudo (não publicado). $\mathrm{O}$ uso de agregado e a proporção deste dependeu das características do efluente a ser tratado. $\mathrm{O}$ uso da policultura possibilitou melhores resultados quando comparado ao experimento realizado em pesque-pague, utilizando-se apenas de aguapé (resultados não divulgados do próprio autor).

O sistema "CWS's" é um processo dinâmico e sua eficiência pode variar com o tempo de tratamento e dependendo do parâmetro estudado do efluente tratado, semelhantemente o que foi verificado por Solano, Soriano e Ciria (2004). O conhecimento prévio da composição do efluente a ser tratado mostrou-se importante para o estabelecimento dos parâmetros a serem analisados, bem como para definição do projeto de construção dos CWS's. Em estudos realizados em paralelo nos reatores 
instalados no CEPTA/IBAMA, Pirassununga, para tratar o efluente dos tanques de criação de $O$. niloticus, foram constatadas variações da eficiência dos CWs durante os 128 dias de tratamento (não publicado).

O uso de solo propicia em alguns parâmetros melhores eficiências, como no início do experimento acarretando remoções de fósforo consideráveis de bom nível. Mostrou-se não adequado para uma rápida remoção de nitrogênio e matéria orgânica, dentro das condições e dimensões aqui utilizadas. O sistema deve ser antes estabilizado para depois ser usado, pois provavelmente o mecanismo da decomposição da matéria orgânica nele presente interfere muito nas análises dos parâmetros aqui determinados. Verificou-se o acréscimo dos valores de amônia que pode ser tóxica em determinadas concentrações, dependendo do valor de $\mathrm{pH}$.

As remoções iniciais elevadas do fósforo pelos tratamentos contendo solo podem não permanecer em períodos prolongados. O solo pode saturar desse nutriente não o removendo mais da fase líquida. Concentrações de ferro e alumínio podem prolongar o tempo de retirada desse nutriente. Estudos mais prolongados são necessários para determinar o efeito da saturação do matriz suporte.

O caco de telha pode apresentar eficiências diferentes conforme sua granulometria. Cacos com "pedaços" maiores possibilitam um menor problema de colmatação (crescimento do biofilme bactéria impedindo a passagem do efluente), enquanto que uma granulometria menor possibilita maior área superficial para crescimento do biofilme. Uma saída para esse dilema seria a adoção de granulometria maior no inicial do sistema de tratamento, seguido por uma granulometria menor no final do sistema.

O uso de solo deve ser evitado por apresentar grande possibilidade de colmatação com o decorrer do tempo, mas sua diminuta granulometria favorece grande superfície para crescimento bacteriano. O uso de solo pode ser substituído por areia (grossa ou média), mas essa matriz suporte apresenta mínimas concentrações de alumino e ferro, diminuindo a possibilidade de remoção de fósforo.

A escolha da matriz suporte depende das características do efluente e qual o destino do mesmo após tratamento.

\section{CONCLUSÕES}

O sistema "CWS's" é um processo dinâmico e sua eficiência pode variar com o tempo de tratamento e dependendo do parâmetro estudado do efluente tratado. A escolha do uso de SC, cacos de tijolos ou proporções entre estes dependem da disponibilidade dos mesmos na região de estudo. Esta possibilitou a fácil aquisição dos cacos de tijolos por situar-se dentro do Pólo Cerâmico de Santa Gertrudes, estado de São Paulo, onde este agregado encontrado como rejeito de olarias. Os resultados demonstraram que os CWS's contendo SC e o agregado apresentaram estabilização do sistema em um período menor em relação aos demais. Após a estabilização de 
todos CWS's, as melhores eficiências foram observadas nos reservatórios contendo apenas cacos de tijolos ou apenas SC, dependendo do parâmetro analisado.

\section{AGRADECIMENTOS}

Os autores agradecem o CEA/UNESP Rio Claro, SP e CEPTA/IBAMA, Pirassununga, SP, Brasil por facilitarem a execução deste estudo e a FAPESP/Processo $n^{\circ}$ 03/08627-0 pelo financiamento deste projeto. Estes também abrangem Eleni Nadai Malagutti, Francisca de Assis Mattioli Gonçalves, Alexandre A.O. Santos e Sara Cristina Galvão.

\section{REFERÊNCIAS}

ANSOLA, G.; GONZÁLEZ, J.M.; CORTIJO, R.; LUIS, E. Experimental and fullscale pilot plant constructed wetlands for municipal wastewaters treatment. Ecological Engineering, Ireland, n. 21, p. 43-52, nov, 2003.

BORGES, K.P. Eficiência de um sistema de áreas alagadas (constructed wetland), para tratamento de águas residuárias. 2001. 112 f. Dissertação (Mestrado em Microbiologia Aplicada) - Instituto de Biociências do Campus de Rio Claro, Universidade Estadual Paulista, Rio Claro.2001.

BRASIL - MINISTÉRIO DO MEIO AMBIENTE. Conselho Nacional do Meio Ambiente - CONAMA. Resoluções CONAMA, no 357. Brasília, DF: SEMA. 2005.23p.www.dicionario.pro.br/.../Resolução_CONAMA_n_357/2005

DUARTE, S. Estudo das potencialidades das zonas húmidas artificiais no tratamento de efluentes aqüícolas. 2002. 31f. Trabalho de Conclusão de Curso (Licenciatura em Engenharia do Ambiente) Instituto Superior Técnico, Departamento de Eng. Biológica e Química, 2002.

ELIAS, J.M. Análise da eficiência global do sistema de "wetlands" construído na estação de tratamento de água para abastecimento público no município de Analândia. 2003. 211f.Dissertação (Mestrado em Conservação e Manejo de Recursos), Programa de Pós Graduação Conservação e Manejo de Recursos - Centro de Estudos Ambientais, Universidade Paulista, Rio Claro. 2003.

GOLTERMAN, H.L.; CLYMO, R.S.; OHNSTAND, M.A. Methods for physical and chemical analysis of fresh water. Oxford: Blackwell Scientific Publications.213p.1978. (IPB Handbook, n.8) 
GOPAL, B. Natural and constructed wetlands for wastewater treatments: potentials and problems. Water Science and Technology, New York, v. 40, n. 3, p. 27-35, 1999.

HAMMER, D. A. Constructed wetlands for wastewater treatment: municipal, industrial and agricultura. Chelsea, Lewis, 350p, 1989.

MACKERETH, F. I. F.; HERON, J.; TALLING, J. F. Water analysis: some revised methods for limnologists. London: Freswater Biological Association, Scientific Publication, n. 36, 1978. 121p.

MICHAEL, J. H. J. Nutrients in salmon hatchery wastewater and its removal through the use of a wetland constructed to treat off-line settling pond effluent. Aquaculture, 226, p 213-225, 2003.

SHUTES, R.B.E. Artificial wetlands and water quality improvement. Environment International, n. 26, p. 441- 447, 2001.

SOLANO, M.L.; SORIANO, P.; CIRIA, M.P. Constructed wetlands as a sustainable solution for wastewater treatment in small villages. Biosystems Engineeering, v. 87, n. 1, p. 109-118, 2004.

STOTTMEISTER, U.; WIEBNER, A.; KUSCHK, P.; KAPPELMEYER, U.; KÄSTNER, M.; BEDERSKI, O.; MÜLLER, R., A.; MOORMANN., H. Effects of plants and microorganisms in constructed wetlands treatment. Biotechnology Advances, n. 22, p. 93-117, 2003.

TANNER, C. C. Plants for constructed wetland treatment systems: A comparison of the growth and nutrient uptake of eight emergent species. Ecological Engineering, Hamilton, v. 7, p. 59-83, 1996.

TODD, J. Ecological engineering, living machines, and the visionary landscape. In: Etnier, C. e Guterstam, B. (eds.), Ecological Engineering for Wastewater Treatment. CRC Press, Inc., Boca Raton, Fl. 1997. p. 113-122.

WALLACE, S. Putting wetlands to work. New York: American Society of Civil Engineers, 1998.

WELSCH, D.J.; SMART, D.L.; BOYER, J.N.; MINKIN, P.; SMITH, H.C.; MCCANDLESS, T.L. Forested Wetlands Functions, Benefits and the Use of Best Management Practies. 1995. 63p. 